\title{
Trastornos de la conducta alimentaria: alteraciones cardiovasculares al ingreso y evolución a 3 meses
}

\author{
VERÓNICA MARÍN B. ${ }^{1}$, VIVIAN RYBERTT ${ }^{1}$, ANA M. BRICEÑO ${ }^{1}$, \\ MARCELA ABUFHELE ${ }^{1}$, PASCUALA DONOSO ${ }^{1, a}$, \\ MACARENA CRUZ ${ }^{1, a}$, ALEJANDRA SILVA ${ }^{1, b}$, \\ CAROLINA PALACIOS ${ }^{1, b}$, LORENA CEA ${ }^{1, b}$, KATERINA SOMMER $^{1,}$ \\ MARIANA LABBÉ
}

\section{Female adolescents with eating disordes: cardiac abnormalities}

Background: Cardiovascular complications can occur in up to $80 \%$ of adolescent patients with eating disorders (ED) and account for $30 \%$ of their mortality. Aim: To evaluate cardiovascular complications in adolescents with ED and their evolution after refeeding. Patients and Methods: In adolescents with ED admitted to treatment, we assessed the nutritional status, weight loss prior to consultation, presence of bradycardia (BC, defined as heart rate $<60$ bpm), we performed an electrocardiogram (ECG) and an echocardiography and measured thyroid hormones. Results: We studied 53 women aged $16.4 \pm$ 2.3 years. Fifteen had a diagnosis of Anorexia Nervosa (AN), seven of Bulimia $(B N)$, eight a not otherwise specified ED (ED-NOS), four a Binge Eating Disorder (BED), sixteen an Atypical Anorexia (AAN) and three an Atypical Bulimia (ABN). Thirty four percent were malnourished and $3.8 \%$ overweight. The most common cardiac problem was BC in 51\%. In eight of 26 patients in whom an echocardiogram was done, it was abnormal. Six had a decreased ventricular mass, three a pericardial effusion and three valvular involvement. There was a significant association between bradycardia and malnutrition, weight loss and low free triiodothyronine levels. BC was significantly more common in patients with AN, but it also occurred in half of the patients with AAN and in one of three patients with other types of ED. At follow up, bradycardia significantly improved with refeeding. Conclusions: There is an association between all types of ED and bradycardia, as well as anatomical and functional cardiac anomalies.

(Rev Med Chile 2019; 147: 47-52)

Key words: Adolescent Medicine; Bradycardia; Cardiovascular Diseases; Feeding and Eating Disorders.

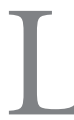

os trastornos alimentarios (TAI) constituyen problemas de salud complejos y preva/entes ${ }^{1}$. En la literatura internacional son la tercera enfermedad crónica en adolescentes, después de la obesidad y asma ${ }^{2}$. En Chile existen datos aislados de prevalencia ${ }^{3,4}$, pero no cifras en adolescentes a nivel nacional. Son trastornos psíquicos con consecuencias orgánicas graves, deriva-

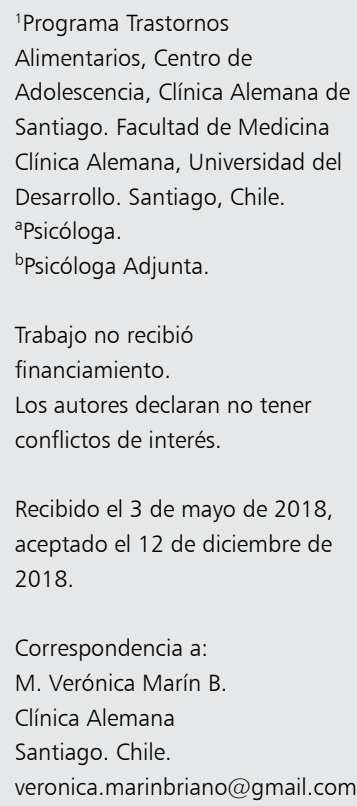

das de la desnutrición, restricciones nutricionales y desequilibrios hidroelectrolíticos, alcanzando una mortalidad de $6 \%$ a $12 \%$ por causas médicas y psiquiátricas $^{5-7}$. La anorexia nerviosa (AN) tiene la mortalidad más alta entre las enfermedades psiquiátricas $^{8,9}$. Las complicaciones cardiovasculares pueden presentarse hasta en $80 \%$ de las pacientes y explican el $30 \%$ de la mortalidad ${ }^{10}$. Estas altera- 
ciones pueden ser tanto estructurales: disminución de masa ventricular, prolapso de válvula mitral o derrame pericárdico; como funcionales: bradicardia sinusal (BC), prolongación del intervalo QTc $\mathrm{y}$ arritmias $^{11}$.

Según un estudio reciente ${ }^{12}$, la prevalencia de $\mathrm{BC}$ es similar en AN y en AN atípica (ANA), cuya única diferencia es que en ANA los pacientes no se encuentran desnutridos.

Las patologías con componente purgativo (vómito autoinducido, abuso de laxantes y diuréticos) también pueden presentar compromiso cardiovascular ${ }^{13}$, por hipokalemia y prolongación del intervalo $\mathrm{QT}^{14}$.

Finalmente, también se han observado complicaciones en pacientes con diagnóstico de TAI con sobrepeso y obesidad, por lo que su búsqueda y detección en estos pacientes también es importante para su tratamiento. El objetivo de este estudio fue evaluar la frecuencia e importancia clínica de las complicaciones cardiovasculares en adolescentes con TAI, las variables asociadas y evolución a 3 meses de tratamiento.

\section{Pacientes y Métodos}

Estudio descriptivo de cohorte, en mujeres de 10 a 24 años, con diagnóstico de TAI DSM-5, ingresadas al Programa de Tratamiento Interdisciplinario de Trastornos Alimentarios, en la Unidad de Adolescencia, Clínica Alemana de Santiago (CAS), desde 2015 a 2017, previo consentimiento escrito e informado de padres/tutores y asentimiento en mayores de 14 años.

Se consideraron criterios de exclusión: ser hombre, presentar otra enfermedad con compromiso orgánico o psiquiátrico relevante; vivir con independencia económica de la familia (nuestro modelo de intervención requiere de la participación activa de los padres/tutores) y presentar consumo problemático de alcohol y drogas.

El diagnóstico de TAI se hizo mediante entrevista clínica psiquiátrica.

Mediante autorreporte o del cuidador se obtuvo: edad, tiempo de evolución, peso previo al inicio del cuadro, pérdida de peso, medicamentos, vómitos y ejercicio físico. Se calculó porcentaje de peso perdido en relación al previo (\% baja de peso) y la velocidad de baja de peso ( $\%$ baja de peso/mes) ${ }^{15}$.

Se evaluó estado nutricional mediante índice de masa corporal (IMC), National Center for Health Statistics/Organización Mundial de Salud (NCHS/OMS) ${ }^{16}$, considerando desnutrición IMC $<$ p10 y sobrepeso IMC $>$ p85 y $<$ p95 para edad y sexo.

La frecuencia cardíaca (FC) y presión arterial (PA) fueron obtenidas en reposo. Bradicardia se definió como $\mathrm{FC}<60$ latidos por minuto (lpm), considerándose de mayor gravedad $<50 \mathrm{lpm}^{17}$ e hipotensión, presión arterial $<90 / 45 \mathrm{mmHg}^{18}$

Además, se evaluaron electrolitos plasmáticos, fósforo, hormonas tiroideas, electrocardiograma en reposo (ECG) y ecocardiograma Doppler. En el ECG, se calculó QTc con fórmula de Bazett, considerándose prolongado $>460$ milisegundos y limítrofe entre 440 y 460 milisegundos ${ }^{19}$. En pacientes con bradicardia se efectuó ecocardiografía transtorácica bidimensional-Doppler-color.

Todas las pacientes recibieron tratamiento según protocolo. Se consideraron criterios para hospitalización: FC $<40 \mathrm{lpm}$, compromiso hemodinámico, riesgo de síndrome de realimentación (SR) (consumir menos de 600 calorías/día; disminución de $1 \mathrm{~kg}$ semanal por 2 semanas o alteraciones hidroelectrolíticas; IMC menor a 70\% del percentil 50) ${ }^{17}$. Las pacientes con FC $>40 \mathrm{y}<50$ lpm sin síntomas que justificaran hospitalización clínica, se trataron en hospitalización domiciliaria. Evaluación por cardiólogo fue requerida en los casos con bradicardia o alteraciones ecocardiográficas.

Se realizó seguimiento y se consignó la evaluación clínica al ingreso, 1 y 3 meses de tratamiento.

Este estudio contó con aprobación del Comité de Ética del Departamento de Investigación de CAS.

\section{Análisis estadístico}

Los resultados son expresados en promedio y desviación estándar o mediana y rango para las variables que no cumplen supuesto de normalidad. Para comparar grupos de muestras independientes se utilizó test $\mathrm{t}$ Student, ANOVA, test Fisher y Mann Whitney. Para comparar evolución en el tiempo se utilizó ANOVA para medidas repetidas $y$ test Q Cochran.

\section{Resultados}

Ingresaron 53 mujeres, $16,4 \pm 2,3$ años (11 a 22 años), con diagnósticos de anorexia nerviosa 
(AN: 15), bulimia (BN: 7), TAI no especificado (TNE: 8), trastorno por atracón (TA: 4), anorexia atípica (ANA: 16) y bulimia atípica (BNA: 3 ). Al ingreso: $34 \%$ desnutridas y $3,8 \%$ sobrepeso; el tiempo de evolución previo al ingreso fue 8,3 meses ( 1 a 39 meses); 12 purgaban con vómitos y 18 compensaban con ejercicio; 9 tomaban fármacos indicados previo al ingreso (8 podrían producir taquicardia y 1 bradicardia); ninguna presentaba alteraciones hidroelectrolíticas ni hipofosfemia; se encontró hormonas tiroideas disminuidas: TSH en 4, T4L en 9 y T3L en 12 pacientes. La complicación más frecuente fue BC, que se presentó en $51 \%$ del total de pacientes y de estas, 33,3\% BC grave. Los principales hallazgos del ECG fueron bradicardia sinusal, sin otras arritmias; el intervalo QTc estaba en rango normal (excepto 1 que fue límite). De las 26 pacientes que tenían ecocardiograma, en 8 estaba alterado: 6 presentaron disminución de masa ventricular, 3 derrame pericárdico y 3 compromiso valvular (1 prolapso válvula mitral y 2 insuficiencia aórtica). Los datos de pérdida de peso previo estaban disponibles en $70 \%$ de las pacientes. El porcentaje baja de peso fue $15,2 \%$ $(3,3 \%$ a $34 \%)$ y la velocidad (\% baja de peso/mes) de $3,4 \% / \mathrm{m}(0,2$ a $16,7 \% / \mathrm{m})$.

Al comparar las características de ingreso según diagnósticos: AN, ANA y otros (que agrupa BN, TNE, TA y BNA), observamos que el grupo con AN estaban significativamente más desnutridas, y, además, tenían el mayor porcentaje de baja de peso, y eran, junto con las pacientes con ANA, las que menos vomitaban. Respecto a complicaciones cardiovasculares, las pacientes con AN presentan mayor frecuencia de bradicardia y alteraciones del ecocardiograma (AN: 5/11; ANA: $1 / 5$ y otros: $2 / 10$, respectivamente). Cabe destacar que en todos los grupos diagnósticos se observan complicaciones cardiovasculares (Tabla 1).

$\mathrm{Al}$ comparar pacientes que presentaban $\mathrm{BC}$ con las que no, observamos que el grupo con $\mathrm{BC}$ consultan antes, tienen mayor porcentaje de baja de peso y velocidad de baja de peso, menor frecuencia de vómitos y T3L más bajas. En relación a los diagnósticos, la $\mathrm{BC}$ fue significativamente más frecuente en pacientes con AN (Tabla 2).

De las 53 pacientes ingresadas, al momento del análisis, 45 habían completado 3 meses de seguimiento. De estas, la evolución de la bradicardia y la desnutrición mostró mejoría en casi $100 \%$ y $90 \%$ de las pacientes, respectivamente (Tabla 3). Ninguna paciente tuvo episodios documentados de arritmia ventricular o muerte durante el estudio.

Tabla 1. Caracterización y complicaciones cardiovasculares según diagnóstico al ingreso

\begin{tabular}{|lcccc|}
\hline Variables & AN & ANA & Otro & p \\
\hline Edad (años) & $\mathbf{n = 1 5}$ & $\mathbf{n = 1 6}$ & $\mathbf{n = 2 2}$ & $0,015^{*}$ \\
\hline Tiempo de evolución $(\mathrm{m})$ & $16,8 \pm 2,1$ & $15,1 \pm 1,9$ & $17,1 \pm 2,4$ & $\mathrm{~ns}$ \\
\hline Z IMC & $7,5 \pm 5,8$ & $7,1 \pm 4,9$ & $9,9 \pm 9,5$ & $<0,001^{*}$ \\
\hline \% baja de peso & $-2,05 \pm 0,5$ & $-0,65 \pm 0,5$ & $-0,39 \pm 1,1$ & $0,02^{*}$ \\
\hline Velocidad de baja de peso (\% baja de peso/m) & $19 \pm 7,6$ & $12,5 \pm 3,8$ & $12,9 \pm 7,6$ & $\mathrm{~ns}$ \\
\hline Desnutrición & $3,80 \pm 2,3$ & $2,94 \pm 1,9$ & $4,22 \pm 5,4$ & $<0,001^{* *}$ \\
\hline Bradicardia $<60$ lpm & $15(83,3 \%)$ & 0 & $3(16,7 \%)$ & $0,027^{* *}$ \\
\hline ECO alterado & $12(80 \%)$ & $7(44 \%)$ & $8(36 \%)$ & $<0,05^{* *}$ \\
\hline Vómitos & $5 / 11$ & $1 / 5$ & $2 / 10$ & $<0,001^{* *}$ \\
\hline Ejercicio & 1 & 1 & 10 & $\mathrm{~ns}$ \\
\hline Fármacos & $2(11,1 \%)$ & $6(33,3 \%)$ & $10(55,6 \%)$ & $\mathrm{ns}$ \\
\hline T3L disminuida & 2 & 1 & 6 & $\mathrm{~ns}$ \\
\hline T4L disminuida & $7(58,3 \%)$ & $3(25,0 \%)$ & $2(16,7 \%)$ & $\mathrm{ns}$ \\
\hline
\end{tabular}

ns: sin diferencia significativa, *ANOVA y **Test Exacto de Fisher. 
Tabla 2. Comparación de variables entre pacientes con y sin bradicardia al ingreso

\begin{tabular}{|lccc|}
\hline Variables & $\begin{array}{c}\text { CON BC } \\
\mathbf{n = 2 7}\end{array}$ & $\begin{array}{c}\text { SIN BC } \\
\mathbf{n = 2 6}\end{array}$ & p \\
\hline Edad (años) & $15,9 \pm 1,9$ & $17,0 \pm 2,6$ & NS \\
AN & 12 & 3 & $0,03^{*}$ \\
ANA & 7 & 9 & \\
Otro & 8 & 14 & \\
\hline FC & $50 \pm 1,2$ & $75 \pm 1,7$ & $0,000^{*}$ \\
\hline Tiempo de & 4 & 8 & $0,03^{* *}$ \\
evolución (m) & $(1-24)$ & $(2-39)$ & \\
\hline Z IMC & $-1,4 \pm 1,0$ & $-0,4 \pm 0,9$ & $0,0013^{*}$ \\
\hline$\%$ baja de peso & $17,4 \pm 7,2$ & $11,3 \pm 4,5$ & $0,009^{*}$ \\
\hline Velocidad de baja & 3,8 & 1,5 & $0,03^{* *}$ \\
de peso & $(0,5-16,6)$ & $(0,18-5,7)$ & \\
\hline Desnutrición & $14(77,8 \%)$ & $4(22,2 \%)$ & $0,008^{* * *}$ \\
\hline Vómitos & $4(33,3 \%)$ & $8(66,7 \%)$ & NS \\
\hline Ejercicio & $12(50 \%)$ & $12(50 \%)$ & NS \\
\hline Fármacos & $3(37,5 \%)$ & $5(62,5 \%)$ & NS \\
\hline T3 disminuida & $10(83,3 \%)$ & $2(16,7 \%)$ & $0,01 * * *$ \\
\hline T4L disminuida & $8(88,9 \%)$ & $1(11,1 \%)$ & ns \\
\hline
\end{tabular}

NS: sin diferencia significativa, *Test de Student, **Test Mann Whitne $y{ }^{* * *}$ Test Exacto de Fisher.

Tabla 3. Evolución de bradicardia y desnutrición a 3 meses

\begin{tabular}{|lcccc|}
\hline Variables & $\begin{array}{c}\text { Ingreso } \\
\mathbf{n = 4 5}\end{array}$ & $\begin{array}{c}\mathbf{1} \text { mes } \\
\mathbf{n}=\mathbf{4 5}\end{array}$ & $\begin{array}{c}\mathbf{3} \text { meses } \\
\mathbf{n = 4 5}\end{array}$ & $\mathbf{p}$ \\
Z IMC & $-1,1 \pm 1,0$ & $-1,0 \pm 0,9$ & $-0,6 \pm 0,7$ & $0,000^{*}$ \\
Desnutrición $17(37,8 \%)$ & $12(26,7 \%)$ & $5(11,1 \%)$ & $0,000^{* *}$ \\
Bradicardia & $25(55,6 \%)$ & $9(20,0 \%)$ & $2(4,4 \%)$ & $0,000 * *$ \\
\hline
\end{tabular}

*ANOVA v. ${ }^{* *}$ test Q Cochran.

En relación al lugar de tratamiento, 5 requirieron hospitalización, todas con AN, y el motivo fue bradicardia y riesgo de SR. Por otro lado, 9 pacientes requirieron hospitalización domiciliaria por la BC.

\section{Discusión}

Las arritmias, especialmente la bradicardia sinusal, son las alteraciones cardíacas más frecuen- tes en los pacientes con TAI ${ }^{9,20,21}$. La mayoría son asintomáticas, incluso en presencia de bradicardia, a menos que esté asociado con hipotensión u otras arritmias. Sin embargo, en pacientes asintomáticos, la bradicardia puede ser un precursor de una arritmia potencialmente letal ${ }^{22}$.

Los mecanismos propuestos para la BC son: aumento del tono vagal, adaptación para conservar energía, pérdida de electrolitos, disminución del contenido de glucógeno en la célula cardiaca, atrofia miofibrilar, edema intersticial, tumefacción mitocondrial y activación de proteinasas dependientes del calcio ${ }^{23}$.

Nuestro estudio, a diferencia de otros que mostraban igual prevalencia de bradicardia en pacientes con $\mathrm{AN}$ vs $\mathrm{ANA}^{10}$, y entre AN y TAI restrictivos ${ }^{24}$, mostró una prevalencia significativamente mayor en las pacientes con AN en relación a la ANA y los otros diagnósticos de TAI. Nuestra hipótesis es que esta diferencia se explicaría porque pacientes con AN presentaban, como es de esperar, mayor desnutrición y, además, mayor porcentaje de baja de peso, siendo ambos los principales factores de riesgo cardiovascular ${ }^{25}$. En relación a la importancia de la baja de peso, por sobre la desnutrición, en estas complicaciones, 2 estudios lo demostraron; uno que revisó 208 niños de 12 años con TAI de reciente diagnóstico, informa que $60 \%$ presentaba bradicardia y $54 \%$ hipotensión, y de estos, solo $59 \%$ estaba desnutrido y $52 \%$ requirió hospitalización al momento del diagnóstico ${ }^{26} \mathrm{y}$ otro, en 161 niños de 13 años de edad, mostraba serias complicaciones cardiovasculares atribuibles a la baja ingesta calórica, la baja de peso, ya que no estaban desnutridos ${ }^{27}$.

Cabe destacar que en nuestro estudio, si bien el mayor riesgo lo presentan las pacientes con AN, esta complicación ocurrió en casi la mitad de las pacientes con ANA y en un tercio de aquellas con otros diagnósticos, dato clínicamente relevante en la evaluación y tratamiento. Al comparar pacientes que presentaban $\mathrm{BC}$ con las que no, se observaron asociaciones ya descritas con desnutrición, mayor porcentaje y velocidad de baja de peso y, además, una asociación con T3L baja, hallazgo también descrito en el estudio de Kastner ${ }^{31}$.

En referencia a alteraciones estructurales encontramos, al igual que otros ${ }^{28,29}$, que 4 de 15 pacientes con AN presentaron disminución de masa ventricular, pero también en 1 de las pacientes con ANA y 1 con TNE, hecho poco comentado 
en las publicaciones. El derrame pericárdico se presentó con menor frecuencia de lo reportado en la literatura ${ }^{30,31}, 2$ de $15(13 \%)$ pacientes con AN y en 1 de $8(12,5 \%)$ de las pacientes con TNE, que independientemente del diagnóstico corresponden a 3 de las 5 pacientes más severamente desnutridas. Además, las 3 pacientes que presentaron compromiso valvular, una con $\mathrm{AN}$ y dos con TNE, todas con desnutrición grave, corresponde a la relación con la disminución de IMC observada previamente $^{29}$.

La rápida evolución a la mejoría de estas alteraciones, con la realimentación y mejoría del estado nutricional, es similar a lo reportado previamente ${ }^{32}$. Como limitación de este estudio, destaca el no contar con el $100 \%$ de los datos del total de pacientes, por lo que no pudimos efectuar análisis para determinar qué variable explica mejor la bradicardia.

En conclusión, los resultados de este estudio respaldan la asociación entre todos los TAI y la bradicardia, además de anomalías cardiacas anatómicas y funcionales.

Finalmente, se refuerza la importancia de la evaluación cardiovascular en el contexto de los TAI, independientemente del diagnóstico nutricional, considerado importantísimo conocer el porcentaje de baja de peso y, si está disponible, la trayectoria individual del IMC, para determinar si ha ocurrido desviación significativa.

Agradecimientos: Agradecemos a la Dra. María Valeria Acevedo Arangua, pediatra cardióloga, CAS, quien ha dado un importante apoyo en el manejo de nuestras pacientes con compromiso cardiovascular.

\section{Referencias}

1. Campbell K, Peebles R. Eating Disorders in Children and Adolescents: State of the Art. Review Pediatrics 2014; 134 (3): 582-92.

2. González A, Cohn MR, Clarke SD. Eating disorders in adolescents. Aust Fam Pfysician 2007; 36: 614-9.

3. Vicente B, Kohn R, Rioseco P, Saldivia S, Baker C, Torres $\mathrm{S}$. Population prevalence of psychiatric disorders in Chile: 6-month and 1-month rates. Br J Psychiatry 2004; 184: 299-305.

4. Granillo MT, Grogan-Kaylor A, Delva J, Castillo M. Eating Disorders Among a Community-Based Sample of Chilean Female Adolescents. J Res Adolesc 2011; 21 (4): 762-8.

5. Fichter MM, Quadflieg N. Mortality in eating disorders results of a large prospective clinical longitudinal study. Int J Eat Disord 2016; 49 (4): 391-401.

6. Debra LF, Aparna K, Kamryn TE, Meera K, Martha $\mathrm{CD}$, Pamela $\mathrm{K}$, et al. A Longitudinal Investigation of Mortality in Anorexia Nervosa and Bulimia Nervosa. Am J Psychiatry 2013; 170: 917-25.

7. Arcelus J, Mitchell A, Wales J, Nielsen S. Is there an elevated mortality rate in anorexia nervosa and other eating disorders? A meta-analysis of 36 studies. Arch Gen Psychiatry 2011; 68: 724-31.

8. Di Cola G, Jacoangeli F, Jacoangeli F, Lombardo M, Iellamo F. Cardiovascular disorders in anorexia nervosa and potential therapeutic targets. Intern Emerg Med 2014; 9: 717-21.

9. Nahshoni E, Weizman A, Yaroslavsky A, Toledano A, Sulkes J, Stein D. Alterations in QT dispersion in the surface electrocardiogram of female adolescents diagnosed with restricting-type anorexia nervosa. J Psychosom Res 2007; 62: 469-72.

10. Morris R, Prasad A, Asaro J, Guzmán M, Sanders L, Hauck A, et al. Markers of Cardiovascular Dysfunction in Adolescents With Anorexia Nervosa. Global Pediatric Health 2017; 4: 1-4.

11. Guerrier K, Mitan L, Wang Y, Czosek RJ. Risk for prolonged QT interval and associated outcomes in children with early restrictive eating patterns. Cardiol Young 2016; 26 (4): 644-9.

12. Sawyer SM, Whitelaw M, Le Grange D, Yeo M, Hughes EK. Physical and Psychological Morbidity in Adolescents With Atypical Anorexia Nervosa. Pediatrics 2016; 137 (4): e20154080.

13. Forney KJ, Buchman-Schmitt JM, Keel PK, Frank GKW. The medical complications associated with purging. Int J Eat Disord 2016; 49 (3): 249-59.

14. Buchanan R, Ngwira J, Amsha K. Prolonged QT interval in bulimia nervosa. BMJ Case Rep 2011; 2011: bcr0120113780.

15. Peebles R, Hardy KK, Wilson JL, Lock JD. Are Diagnostic Criteria for Eating Disorders Markers of Medical Severity? Pediatrics 2010; 125 (5): e1193-201.

16. https://www.cdc.gov/growthcharts/clinical_charts.htm

17. The Royal Colleges of Psychiatrists. Summary of Junior MARSIPAN: Management of Really Sick Patients under 18 with Anorexia Nervosa. Coll Rep 2015; 168s.

18. Golden NH, Katzman DK, Sawyer SM, Ornstein RM, Rome ES, Garber AK, et al. Update on the medical management of eating disorders in adolescents. J Adolesc Health 2015; 56 (4): 370-5. 
19. Goldenberg I, Moss AJ, Zareba W. QT interval: How to measure it and what is "normal". J Cardiovasc Electrophysiol 2006; 17 (3): 333-6.

20. Sachs KV, Harnke B, Mehler PS, Krantz MJ. Cardiovascular complications of anorexia nervosa: A systematic review. Int J Eat Disord 2016; 49 (3): 238-48.

21. Portilla MG. Bradycardia: an important physical finding in anorexia nervosa. J Ark Med Soc 2011; 107 (10): 206-20.

22. Yahalom M, Spitz M, Sandler L, Heno N, Roguin N, Turgeman $\mathrm{Y}$. The significance of bradycardia in anorexia nervosa. Int J Angiol 2013; 22 (2): 83-94.

23. Casiero D, Frishman WH. Cardiovascular complications of eating disorders. Cardiol Rev 2006; 14: 227-31.

24. Le Grange D, Crosby RD, Engel SG, Cao L, Ndungu A, Crow SJ, et al. DSM-IV Defined anorexia nervosa versus subthreshold anorexia nervosa (EDNOS-AN). Eur Eat Disord Rev 2013; 21: 1-7.

25. Whitelaw M, Gilbertson H, Lee KJ, Sawyer SM. Restrictive eating disorders among adolescent inpatients. Pediatrics 2014; 134: e758-64.

26. Hudson LD, Nicholls DE, Lynn RM, Viner RM. Medical instability and growth of children and adolescents with early onset eating disorders. Arch Dis Child 2012; 97 (9): 779-84.

27. Pinhas L, Morris A, Crosby RD, Katzman DK. Incidence and age-specific presentation of restrictive eating disorders in children: a Canadian Paediatric Surveillance Program study. Arch Pediatr Adolesc Med 2011; 165 (10): 895-9.

28. Escudero CA, Potts JE, Lam PY, De Souza AM, Mugford GJ, Sandor GG. An Echocardiographic Study of Left Ventricular Size and Cardiac Function in Adolescent Females with Anorexia Nervosa. Eur Eat Disord Rev 2016; 24 (1): 26-33.

29. Oflaz S, Yucel B, Oz F, Sahin D, Ozturk N, Yaci O, et al. Assessment of myocardial damage by cardiac MRI in patients with anorexia nervosa. Int J Eat Disord 2013; 46 (8): 862e.

30. Kastner S, Salbach-Andrae H, Renneberg B, Pfeiffer E, Lehmkuhl U, Schmitz L. Echocardiographic findings in adolescents with anorexia nervosa at beginning of treatment and after weight recovery. Eur Child Adolesc Psychiatry 2012; 21: 15-21.

31. Ramacciotti CE, Coli E, Biadi O, Dell'Osso L. Silent pericardial effusion in a sample of anorexic patients. Eating Weight Disord 2003; 8: 68-713.

32. Shamim T, Golden NH, Arden M, Filiberto L, Shenker IR. Resolution of vital sign instability: An objective measure of medical stability in anorexia nervosa. J Adolesc Health 2003; 32: 73-7. 